\title{
Iontoforesis transdérmica con dexametasona y verapamilo para la Enfermedad de La Peyronie
}

\author{
R. Cabello Benavente, I. Moncada Iribarren, A. de Palacio España, A. Hernández Villaverde, \\ J.I. Monzó, C. Hernández Fernández
}

Hospital General Universitario Gregorio Marañón. Madrid.

Actas Urol Esp 2005; 29 (10): 955-960

\begin{abstract}
RESUMEN
IONTOFORESIS TRANSDÉRMICA CON DEXAMETASONA Y VERAPAMILO PARA LA ENFERMEDAD DE LA PEYRONIE

Objetivo: Evaluar los efectos de la administración transdérmica iontoforética de verapamilo y dexametasona en pacientes con enfermedad de La Peyronie de menos de un año de evolución.

Material y métodos: Tratamos a diez pacientes, dos veces a la semana durante seis semanas consecutivas, mediante un dispositivo Miniphysionizer@. Este sistema genera una corriente de $2 \mathrm{~mA}$ durante un ciclo de 20 min, que consigue la penetración transdérmica de la medicación. En cada sesión se administró dexametasona 8 mg y verapamilo 5 mg en un pequeño depósito autoadhesivo, pegado en la piel del pene, próximo a la placa de fibrosis. Para evaluar la eficacia del tratamiento, medimos la curvatura por el test de Kelami, y delimitamos el tamaño de la placa mediante ecografía peneana. Otros parámetros, como el dolor, la función eréctil y la capacidad para la penetración vaginal se objetivaron mediante un cuestionario. Vigilamos efectos secundarios durante el tratamiento.

Resultados: No se evidenció mejoría en la desviación peneana en ninguno de los pacientes aunque tampoco hubo progresión de la misma. La consistencia de la placa de fibrosis disminuyó en 5 pacientes, llegando a no ser palpable en 2 de ellos. Se objetivó disminución del volumen medido por ecografia en 6 . El dolor mejoró en 8 pacientes, llegando a desaparecer en 6 de ellos. Un paciente manifestó mejoría de la función eréctil al finalizar el tratamiento, mientras que 3 refirieron mejoría en su capacidad para la penetración y 2 que el tratamiento mejoró su vida sexual en general. No objetivamos efectos secundarios destacables, excepto un leve y transitorio eritema cutáneo en el lugar de colocación de los electrodos.

Conclusión: La iontoforesis transdérmica es un tratamiento que parece eficaz para el control del dolor en los estadios iniciales de la enfermedad de La Peyronie. Su eficacia en la mejoría de la curvatura peneana parece limitada. Son necesarios ensayos clínicos controlados y tal vez revisión de sus indicaciones para conseguir efectos clínicos más relevantes.
\end{abstract}

Palabras clave: Iontoforesis. Enfermedad de La Peyronie. Induración peneana.

\section{ABSTRACT}

TRANSDERMAL IONTOPHORESIS WITH DEXAMETHASONE AND VERAPAMIL FOR PEYRONIE'S DISEASE

Objetives: To evaluate the effects of transdermal iontophoresis with verapamil and dexamethasone in patients with Peyronie's disease of less than one year of evolution.

Material and methods: We have treated ten patients twice a week during six consecutive weeks using iontophoresis with a Miniphysionizer@ dispositive. This device generates a $2 \mathrm{~mA}$ electric current during 20 min which triggers the transdermal penetration of medication. In every session dexamethasone $8 \mathrm{mg}$ and verapamil $5 \mathrm{mg}$ were administered inside a small self-adhesive receptacle on the penile skin overlying the fibrosis plaque. To evaluate the efficacy, penile curvature was measured by Kelami's test, while the plaque size was assessed by penile ultrasound. Other parameters like pain, erectile function and ability for vaginal intercourse were recorded using questionnaires. Safety parameters were also assessed during treatment.

Results: No improvement or progression in penile curvature was evidenced in any of the patients. The hardness of the plaque was reduced in 5 patients, becoming impalpable in 2 of them. Decrease in plaque volume was observed by penile ultrasound in 6. Pain improved in 8 patients, disappearing in 6 of them. One patient recovered his erectile function at the end of the treatment; whereas 3 referred that their ability for intercourse enhanced while 2 reported that treatment improved their sexual life in general. We didn't record any significantly side effects, except for a transitory and slight dermal redness on the site of electrode placement.

Conclusions: Transdermal iontophoresis is an effective treatment for pain control in early stages of Peyronie's disease. Efficacy in reducing penile curvature seems to be limited. Controlled clinical trials are needed, and perhaps reviewing indications in order to obtain more relevant clinical effects.

Keywords: Iontophoresis. Peyronie’s disease. Penile induration. 
$\mathrm{L}$ a enfermedad de La Peyronie consiste en una induración fibrosa de los cuerpos cavernosos del pene de causa desconocida. La deformidad peneana adquirida (ya sea curvatura, acuñamiento, deformidad en reloj de arena o acortamiento), es evidente durante la erección ${ }^{1}$. Habitualmente acompañada de induración palpable (placa) en el pene con o sin erección dolorosa $^{1}$. Se le calcula una prevalencia en torno al 0,4-1\% de la población, en aumento desde la introducción de fármacos potenciadores de la erección ${ }^{2}$.

Provoca un conjunto de síntomas fruto de alteraciones estructurales en los cuerpos cavernosos. En la fase inicial es típica la presencia de un nódulo o placa palpable, erecciones dolorosas y/o deformidad peneana con la erección. Síntomas tardíos son una placa fibrosa, la estabilización de la deformidad durante la erección y la disfunción eréctil ${ }^{1}$. La pérdida de elasticidad en el lugar de la placa provoca desviación y/o deformación en el pene, y puede interferir con la oclusión venosa responsable de la erección ${ }^{3}$.

Mientras que la corrección quirúrgica es el tratamiento de elección en la fase final de la enfermedad y en los casos de mayor deformidad, se han probado una gran variedad de tratamientos médicos para intentar resolver los síntomas. La limitación de la medicación oral (vitamina E, tamoxifeno, colchicina, aminobenzoato potásico, interferon, etc.) por sus efectos secundarios y/o su escasa respuesta ha obligado a buscar otras vías para la administración de medicación. La difusión transdérmica de medicación se ha propuesto como alternativa para la administración de medicación en el interior de la albugínea ${ }^{4}$. Han sido publicados prometedores estudios sobre su utilidad en la enfermedad de La Peyronie P,5-7. $^{3,}$

\section{OBJETIVO}

Evaluar los efectos de la administración transdérmica iontoforética de dexametasona y verapamilo en la enfermedad de La Peyronie, respecto a la desviación, el volumen de la placa, el dolor con la erección, la función eréctil y la capacidad para la penetración en pacientes en la fase inicial de la enfermedad (menos de un año de evolución).

\section{MATERIAL Y MÉTODOS}

Entre Junio y Noviembre de 2004, se reclutaron diez pacientes consecutivos que consultaron por sintomas compatibles con la enfermedad de La Peyronie. Fueron informados sobre el tratamiento y firmaron un consentimiento específico, diseñado y aprobado por el Comité Ético de Investigación Clínica del hospital.

Con edades comprendidas entre 24 a 65 años (media de 48). El tiempo desde la presentación de la enfermedad fue menor de 3 meses en un paciente, de 3 a 6 meses en 7, y de 6 a 12 meses en 2. Definian el comienzo como súbito 5 pacientes y gradual el resto. La forma de presentación más frecuente fue la triada conjunta de dolor con la erección, desviación y la aparición de una placa palpable. La localización de la placa de fibrosis más frecuente fue en la región dorsal $(n=8)$, y la desviación observada más frecuente fue también dorsal $(n=7)$. Sólo 3 pacientes referían un traumatismo peneano previo. Encontramos un paciente con el antecedente de enfermedad de La Peyronie en su familia y dos presentaban además signos de enfermedad de Dupuytren.

Ningún paciente había recibido tratamiento antes de comenzar con las sesiones de iontoforesis. Se analizaron los antecedentes personales de los pacientes en busca de criterios de exclusión (tratamiento reciente y/o alergia a antagonistas del calcio o corticoides, presencia de disfunción eréctil de otra etiología, portador de marcapasos), datos sobre el inicio de la enfermedad y síntomas observados. No se encontraron antecedentes urológicos de interés.

Fueron tratados dos veces a la semana durante seis semanas consecutivas. El tratamiento consistió en la administración de la medicación (dexametasona $8 \mathrm{mg}$ y verapamilo $5 \mathrm{mg}$ ) dentro de un depósito autoadhesivo desechable (ánodo) (Fig. 1), pegado a la piel del pene sobre la placa de fibrosis. El depósito se conecta a un dispositivo EMDA (electromotive drug administration) Miniphysionizer@ (modelo MP 2.0, Physion, Italy) (Fig. 2). El sistema tiene a su vez un electrodo dispersivo (cátodo), que una vez humedecido y aplicado en la piel del paciente (muslo o hipogastrio), cierra el circuito (Fig. 3). El sistema EMDA Miniphysionizer@, genera una corriente de $2 \mathrm{~mA}$ con $2500 \mathrm{~Hz}$. durante un ciclo de $20 \mathrm{~min}$. 


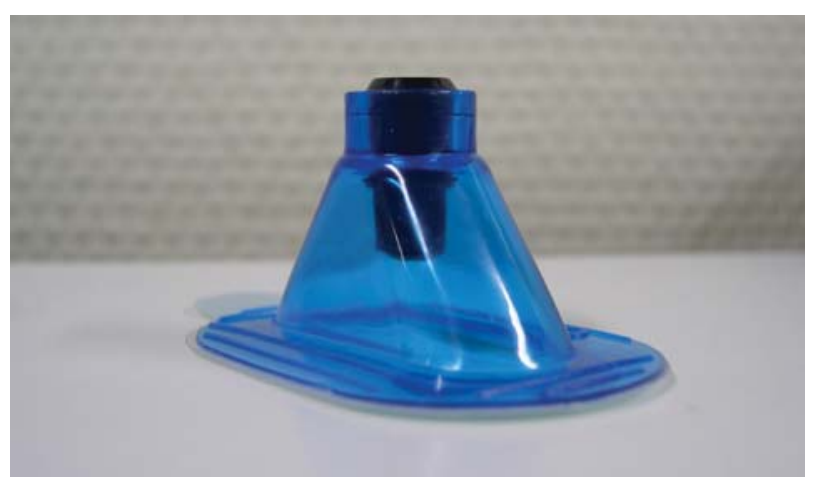

FIGURA 1

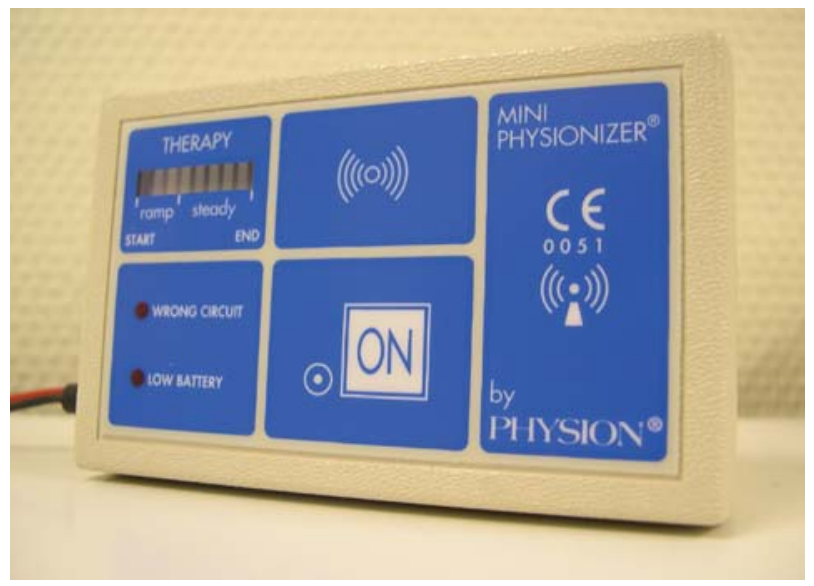

FIGURA 2

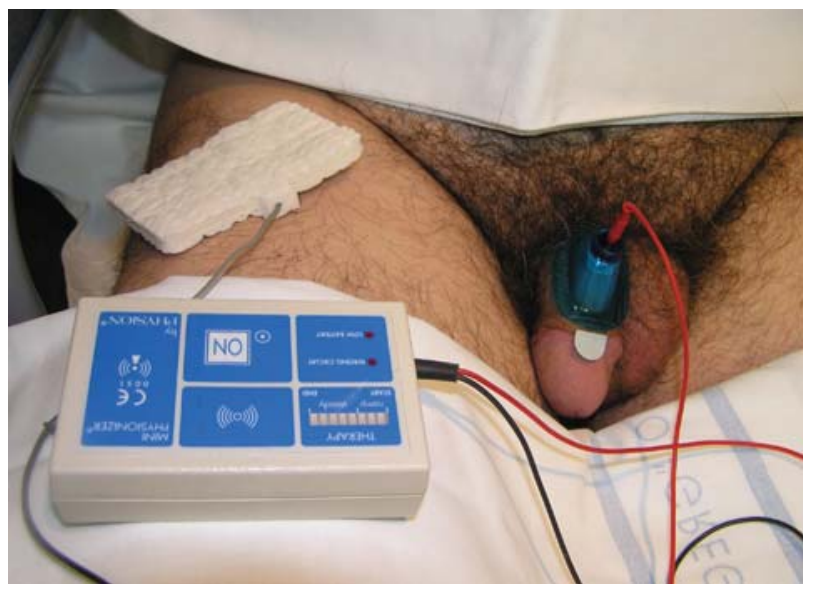

FIGURA 3

Se obtuvieron datos objetivos antes de comenzar el tratamiento, así como al mes y a los seis meses una vez concluido este. El tamaño de la placa se evaluó mediante ecografía peneana, la desviación mediante el test de Kelami ${ }^{8}$ (autofotografias en tres planos en erección completa o tras administración de IIC de PGE2 20 mg). La consistencia y localización mediante exploración física.
La función eréctil a través de los items 1 a 5 y 15 del "International Index of Erectile Function" $(\mathrm{IIEF})^{9}$. El dolor y la afectación sobre la vida sexual en general se objetivaron mediante un cuestionario.

Durante el tratamiento se vigilaron efectos secundarios y se monitorizó la tensión arterial. Todos los pacientes completaron las doce sesiones programadas.

\section{RESULTADOS}

No se evidenciaron cambios en la evolución de la enfermedad en las dos revisiones realizadas (al mes y a los seis meses de finalizar el tratamiento). La placa se etiquetó como fibrosa $(n=6)$ o elástica $(\mathrm{n}=4)$ al inicio del tratamiento. Disminuyendo de consistencia en 5 pacientes ( 3 previamente fibrosa y 2 previamente elástica), llegando a no ser palpable en 2 de ellos. Se objetivó reducción del volumen medido por ecografia de la placa en 6 pacientes (Tabla 1 ).

La desviación objetivada por el test de Kelami ${ }^{8}$ antes del tratamiento, se clasificó en $<30^{\circ}(n=5)$, de $30-60^{\circ}(\mathrm{n}=5)$ y $>60^{\circ}(\mathrm{n}=0)$. Al finalizar no se evidenció mejoría en la desviación en ningún paciente, aunque tampoco hubo progresión en la misma.

El dolor mejoró en 8 pacientes, llegando a desaparecer en 6 de ellos, lo que en la mayoría ocurrió antes de completar la mitad del tratamiento.

Un paciente manifestó mejoría de la función eréctil al finalizar el tratamiento, 4 pacientes refirieron mejoría de la capacidad de penetración y 6 que había mejorado en general su vida sexual (Tabla 2).

Realizamos una encuesta sobre satisfacción al finalizar el protocolo; 7 pacientes se mostraron satisfechos con los resultados obtenidos, 2 ni satisfechos ni insatisfechos y 1 insatisfecho.

No objetivamos efectos secundarios destacables ni repercusión aparente sobre la tensión arterial. La administración del ciclo resultó indolora. Como único dato llamativo, encontramos un leve y transitorio eritema cutáneo en el lugar de colocación de los electrodos, que aparecía de forma constante al finalizar cada ciclo de tratamiento. Ningún paciente abandonó el estudio. 
Tabla 1

\begin{tabular}{|c|c|c|c|c|c|c|c|c|c|c|}
\hline & \multirow[t]{2}{*}{ Desviación } & \multirow[t]{2}{*}{ Placa } & \multicolumn{2}{|c|}{$\begin{array}{l}\text { Volumen placa } \\
\qquad\left(\mathrm{mm}^{3}\right)\end{array}$} & \multicolumn{2}{|c|}{ Consistencia placa } & \multicolumn{2}{|c|}{ Kelami } & \multicolumn{2}{|c|}{$\begin{array}{c}\text { Dolor } \\
\text { Erección }\end{array}$} \\
\hline & & & Pre & Post & Pre & Post & Pre & Post & Pre & Post \\
\hline 1 & Dorsal & Proximal & 189 & 98 & Fibrosa & Elástica & $<30^{\circ}$ & $<30^{\circ}$ & Leve & Ausente \\
\hline 2 & Dorsal & Proximal & 276 & 86 & Elástica & Elástica & $<30^{\circ}$ & $<30^{\circ}$ & Moderado & Leve \\
\hline 3 & Lateral & Medio & 297 & 90 & Fibrosa & Elástica & $30-60^{\circ}$ & $30-60^{\circ}$ & Moderado & Ausente \\
\hline 4 & Lateral & Distal & 242 & 140 & Elástica & Elástica & $30-60^{\circ}$ & $30-60^{\circ}$ & Moderado & Ausente \\
\hline 5 & Dorsal & Proximal & 144 & 140 & Fibrosa & Fibrosa & $30-60^{\circ}$ & $30-60^{\circ}$ & Leve & Ausente \\
\hline 6 & Lateral & Distal & 570 & 144 & Elástica & No palpable & $<30^{\circ}$ & $<30^{\circ}$ & Leve & Ausente \\
\hline 7 & Dorsal & Distal & 80 & 77 & Fibrosa & Elástica & $<30^{\circ}$ & $<30^{\circ}$ & Moderado & Moderado \\
\hline 8 & Dorsal & Proximal & 98 & 97 & Fibrosa & Fibrosa & $30-60^{\circ}$ & $30-60^{\circ}$ & Leve & Ausente \\
\hline 9 & Dorsal & Medio & 249 & 89 & Elástica & No palpable & $30-60^{\circ}$ & $30-60^{\circ}$ & Severo & Leve \\
\hline 10 & Dorsal & Medio & 196 & 200 & Fibrosa & Fibrosa & $<30^{\circ}$ & $<30^{\circ}$ & Leve & Leve \\
\hline
\end{tabular}

Desviación; objetivada mediante test de Kelami. Placa; localización según proximal/medio/distal. Volumen placa; medido mediante ECO peneana. Consistencia placa; fibrosa/elástica. Kelami; grado de desviación, clasificado según $<30^{\circ} / 30-60^{\circ} />60^{\circ}$. Dolor erección; dolor expresado por los pacientes sobre escala analógica (0 a 10) y estratificado (Ausente; 0, Leve; 1 a 3, Moderado; 4 a 7 , Severo; 8 a 10).

Tabla 2

\begin{tabular}{cccccc}
\hline & Función eréctil & \multicolumn{2}{c}{$\begin{array}{c}\text { Capacidad } \\
\text { penetración }\end{array}$} & $\begin{array}{c}\text { Mejoria } \\
\text { vida } \\
\text { sexual } \\
\text { Post }\end{array}$ \\
\hline 1 & Pre & Post & Pre & Post & Sí \\
2 & $>25$ & $>25$ & Difícil & Mejoría & Sí \\
3 & $17-25$ & $17-25$ & Difícil & No Mejoría & No \\
4 & $11-16$ & $11-16$ & Difícil & No Mejoría & No \\
5 & $17-25$ & $17-25$ & Difícil & No Mejoría & No \\
6 & $17-25$ & $>25$ & Posible & Mejoría & Sí \\
7 & $17-25$ & $17-25$ & Difícil & No Mejoría & Sí \\
8 & $>25$ & $>25$ & Posible & Mejoría & Sí \\
9 & $>25$ & $>25$ & Difícil & Mejoría & No \\
10 & $17-25$ & $17-25$ & Difícil & No mejoría & Sí \\
\hline
\end{tabular}

Función eréctil; evaluada mediante los ítems 1 a 5 y 15 del IIEF (9)

\section{DISCUSIÓN}

La fisiopatología y el curso de la enfermedad de La Peyronie siguen sin conocerse por completo $^{3,6}$. Se ha demostrado mejoría espontánea entre un 7 y un $29 \%$ de los pacientes ${ }^{5}$. En su origen se supone una cicatrización aberrante en un individuo susceptible, junto con un traumatismo con el pene erecto, que provoca una delaminación o sangrado bajo la túnica albuginea ${ }^{1}$. Se ha relacionado con otras patologías como la enfermedad de Dupuytren, la contractura de la fascia plantar, la timpanoesclerosis, la instrumentación uretral, la diabetes, gota, o la enfermedad de Paget, así como con el uso de $\beta$-bloqueantes o fenitoína ${ }^{1,2}$. La edad media de aparición es de 53 años ${ }^{1,2}$.

En conjunto, los tratamientos médicos para la enfermedad de Peyronie no garantizan su resolución, especialmente de la deformidad peneana ${ }^{1}$. Entre la medicación oral y la cirugía, se han investigado otros métodos para administrar medicación al interior de la placa de fibrosis.

El uso de cremas tópicas resulta ineficaz, pues no consiguen que el fármaco difunda a la albúginea ${ }^{10}$. La inyección intraplaca de sustancias (verapamilo $^{11}$, corticoides $^{12}$, colagenasa ${ }^{13}$, orgoteína ${ }^{14}$, interferón ${ }^{15,16}, \beta$-amoniopropionitrilo ${ }^{17}$, etc.) se ha demostrado parcialmente eficaz. Con el importante inconveniente de resultar muy doloroso, en cierta manera invasivo, así como técnicamente difícil, al tratar de inyectar sustancias en el interior del duro tejido de la placa ${ }^{5}$. También se han probado en un intento de fragmentar la placa, fuentes de energía alternativas, como la radioterapia ${ }^{18}$ o la litotricia $^{19}$, con resultados dispares.

La iontoforesis transdérmica es un método para la administración de medicación de forma no invasiva, utilizada en varios campos de la medicina (antiinflamatorios y analgésicos en patología articular, insulina para pacientes diabéticos...) $)^{2}$. Se ha demostrado eficaz para la difusión de medicación en el interior de la albugínea ${ }^{4}$. 
Su principio fundamental consiste, en utilizar energía en forma de corriente eléctrica de baja amplitud, para conducir moléculas cargadas positivamente en el tejido subyacente ${ }^{4}$. Se considera indoloro, seguro y efectivo ${ }^{3,5-7}$. La combinación de fármacos resulta más eficaz que la monoterapia para el tratamiento de la enfermedad de La Peyronie ${ }^{3}$. Se ha probado a administrar dexametasona, verapamilo y lidocaína en diferentes pautas. La más utilizada es la combinación de verapamilo (por su actividad inhibitoria de la secreción y síntesis de matriz extracelular que provoca reducción del colágeno, glucosaminoglicanos y anti-factor $\beta$ de crecimiento transformante) y dexametasona (por sus propiedades anti-inflamatorias). El uso de la lidocaína se recomienda en situaciones con placas especialmente dolorosas ${ }^{3,6,7}$.

En la enfermedad de La Peyronie, el dolor asociado a la erección habitualmente remite con el tiempo ${ }^{1,2}$. Suele ser persistente durante la fase inflamatoria, y aunque habitualmente no es severo puede interferir con la función sexual. Con la iontoforesis se ha reportado mejoría en el 96$100 \%$ de los $\operatorname{casos}^{3,5-7}$. En nuestra experiencia el dolor mejoró hasta en 8 pacientes, haciéndolo además antes de completar la primera mitad del tratamiento. Parece por tanto que el dolor es un síntoma que puede mejorar con este tratamiento cuando se administra de forma precoz.
En los estudios revisados, destaca la mejoría en la curvatura en un rango entre el $37 \%^{3}$ y $88 \%{ }^{7}$. Estos datos chocan con los que hemos observado, donde no objetivamos mejoría en ningún paciente. Se han comunicado disminución del volumen de la placa entre el $53 \%{ }^{3}$ y el 58 $100 \%{ }^{6}$ de los pacientes. En nuestra serie la placa se redujo en 6 de los 10 pacientes.

La función eréctil mejoró entre el $39 \%^{3}$ y el $88 \%{ }^{7}$. Nosotros lo observamos en uno de los diez pacientes que tratamos. La actividad sexual en general también mejoró entre el $44 \%^{3}$, el $73 \% \%^{5}$, nuestro porcentaje se encuentra en el $60 \%$. La mayoría de los pacientes con motivo de la disminución del dolor con la erección.

Respecto a los efectos secundarios encontrados fueron similares a los comunicados en la literatura y se limitaron a un eritema leve en el lugar de contacto de los electrodos con la piel ${ }^{3,6,7}$.

Los resultados enunciados fueron reevaluados a los seis meses de terminar el tratamiento sin encontrar cambios en la actividad de la enfermedad. Nos llama la atención cómo si observando una reducción de la placa en 6 pacientes, no encontráramos mejoría significativa en la erección, y ningún cambio en la curvatura. Desconocemos el motivo por el que aparece esta discrepancia entre la literatura publicada y nuestro estudio (Tabla 3).

Tabla 3

\begin{tabular}{|c|c|c|c|c|c|c|}
\hline & $\mathbf{n}$ & Tratamiento & Dolor & Curvatura & Potencia & Placa \\
\hline H.G.U.G.M. & 10 & $\begin{array}{c}\text { Verapamilo } \\
\text { Dexametasona }\end{array}$ & $80 \%$ & $0 \%$ & $10 \%$ & $60 \%$ \\
\hline \multirow[t]{2}{*}{$\begin{array}{l}\text { Montorsi } \\
\text { J Androl } 2000\end{array}$} & 40 & $\begin{array}{c}\text { Orgoteína } \\
\text { Dexametasona } \\
\text { Lidocaína }\end{array}$ & $100 \%$ & $62 \%$ & $84 \%$ & $79 \%$ \\
\hline & 25 & $\begin{array}{c}\text { Verapamilo } \\
\text { Dexametasona }\end{array}$ & $100 \%$ & $88 \%$ & $88 \%$ & $90 \%$ \\
\hline $\begin{array}{l}\text { Rield } \\
\text { J Urol } 2000\end{array}$ & 100 & $\begin{array}{l}\text { Verapamilo } \\
\text { Dexametasona } \\
\text { (lidocaína) }\end{array}$ & $96 \%$ & $37 \%$ & $39 \%$ & $53 \%$ \\
\hline $\begin{array}{l}\text { Di Stasi } \\
\text { BJU Int } 2003\end{array}$ & 49 & $\begin{array}{c}\text { Verapamilo } \\
\text { Dexametasona }\end{array}$ & $96 \%$ & $84 \%$ & $59 \%$ & $82 \%$ \\
\hline \multirow[t]{2}{*}{$\begin{array}{l}\text { Di Stasi } \\
\text { J Urol } 2004\end{array}$} & 37 & $\begin{array}{c}\text { Verapamilo } \\
\text { Dexametasona }\end{array}$ & $96 \%$ & $57 \%$ & $51 \%$ & $100 \%$ \\
\hline & 36 & Lidocaína & temporal & $11 \%$ & $11 \%$ & $58 \%$ \\
\hline
\end{tabular}

Comparación de los resultados obtenidos con los encontrados en la literatura: Montorsi (J Androl 2000) ${ }^{6}$; estudio doble ciego, controlado con placebo y parcialmente cruzado. Rield (J Urol 2000) ${ }^{3}$; estudio no controlado. Di Stasi (BJU Int 2003) ${ }^{4}$; estudio no controlado. Di Stasi (J Urol 2004) ${ }^{5}$; estudio controlado. 
Solamente la realización de estudios controlados y el seguimiento a largo plazo, serán capaces de demostrar si la iontoforesis es capaz de frenar la evolución de la enfermedad, reduciendo la necesidad de cirugía.

\section{CONCLUSIONES}

La iontoforesis transdérmica es un tratamiento seguro y no invasivo, que parece eficaz para el control del dolor en los estadios iniciales de la enfermedad de La Peyronie. Su eficacia en la mejoría de la curvatura peneana parece limitada.

En nuestra experiencia, realizada en un grupo pequeño de pacientes y sin grupo de control, creemos que se precisan de más ensayos controlados para comprobar si los efectos encontrados en la reducción del volumen de la placa se corresponden con mejorías en la curvatura peneana, y para confirmar que la mejoría en el control del dolor, y la función sexual se mantienen en el tiempo. Ensayos controlados podrían a su vez ayudar a establecer la pauta de tratamiento más adecuada para estos pacientes.

\section{REFERENCIAS}

1. Gholami SS, Gonzalez-Cadavid NF, Lin C-S. Rajfer J, Lue TF. Peyronie's disease: a review. J Urol 2003;169:12341241 .

2. Lewis RW, Jordan GH. Surgery for erectile dysfunction. En Walsh P. C et al Editors. "Campbell's urology, eighth edition”. Saunders, Philadelphia. 2002. pp. 1696-1705.

3. Rield C. R., Plas E., Engelhardt P., et al. "Iontophoresis for treatment of peyronie's disease”. J Urol 2000;163:95-99.

3. Levine LA, Estrada CR, Shou W, Cole, A. Tunica albuginea tissue analysis after electromotive drug administration". J Urol 2003;169:1775-1778.

4. Di Stasi SM, Giannantoni A, Capelli G, Jannin EA, Virgili G, Storti L, Vespasiani G. Transdermal electromotive administration of verapamil and dexamethasone for Peyronie's disease. BJU Int 2003;91:825-829.

5. Di Stasi SM, Giannantoni A, Stephen RL, Capelli G, Giurioli, A. Jannini E, Vespasiani G. A prospective randomized study using transdermal electromotive administration of verapamil and dexamethasone for Peyronie's disease. J Urol 2004;171:1605-1608.
6. Montorsi F, Salonia A, Guazzoni G, Barbieri L, Colombo R, Brausi M, Scattoni V, Rigatti P, Pizzini G. Transdermal electromotive multi-drug administration for Peyronie's disease: preliminary results. J Androl 2000;21:85-90.

7. Kelami A. Autophotography in evaluation of functional penile disorders. Urol 1983;21:628-629.

8. Rosen RC, Riley A, Wagner G, Osterloh IH, Kirkpatrick J, Mishra A. The international index of erectile function (IIEF): a multidimensional scale for assessment of erectile dysfunction”. Urol 1997;49:822-830.

9. Martin DJ, Badwan K, Paker M, Mulhall JP. Transdermal application of verapamil gel to the penile shaft fails to infiltrate the tunica albuginea”. J Urol 2002;168(6):2483-2485.

10. Levine LA, Goldman KE, Greenfield J M. Experience with intraplaque injection of verapamil for Peyronie's disease. $J$ Urol 2002;168:621-626.

11. Cipollone G; Nicolai M; Mastroprimiano G; Iantorno R; Longeri D; Tenaglia R. Betamethasone versus placebo in Peyronie's disease. Arch Ital Urol Androl 1998;70(4):165168.

12. Gelbard MK, James K, Riach P, Dorey F. Collagenase versus placebo in treatment of Peyronie's disease: a doubleblind study. J Urol 1993;149:56-58.

13. Primus G. Orgotein in the treatment of plastic induration of the penis (Peyronie's disease). Int Urol Nephrol 1993;25: 169-172.

14. Wegner HE, Andresen R, Knispel HH, Miller K. Local interferon-alpha $2 \mathrm{~b}$ is not an effective treatment in early-stage Peyronie's disease. Eur Urol 1997;32(2):190-193.

15. Polat O, Gul O, Orbey I, et al. Peyronie's disease; intralesional treatment with interferon alpha-2A and evaluation of the results by magnetic resonance imaging. Int Urol Nephrol 1997;29:465-471.

16. Gelbard M, Lindner A, Chvapil M, Kaufman J. Topical beta-aminopropionitrile in the treatment of Peyronie's disease. J Urol 1983;129(4):746-748.

17. Incrocci L, Wijnmaalen A, Slob AK, Hop WCJ, Levendag PC. Low dose radiotherapy in 179 patients with Peyronie's disease: treatment outcome and current sexual functioning. Int J Radiat Oncol Biol Phys 2000;47:1353-1356.

18. Lebret T, Herve JM, Lugagne PM, et al. Extra-corporeal lithotripsy in treatment of Peyronie's disease. Use of standard lithotriptor (Multiline Siemens) on "young" (less then six months old) plaques. Prog Urol 2000;10:65-71.

Dr. R. Cabello Benavente

Avda. Galaxia 6, esc. 2, $2^{\circ}$ b.

28023 Madrid

E-mail: ramirocabello@telefonica.net

(Trabajo recibido el 30 de junio de 2005) 